\title{
Electromagnetic Transitions of Hyperons in a Relativistic Quark Model
}

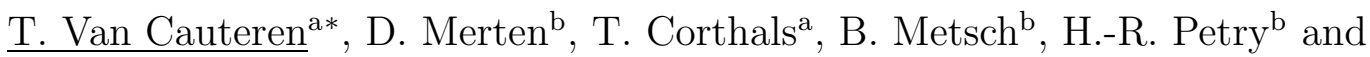

J. Ryckebusch ${ }^{\mathrm{a}}$

${ }^{a}$ Department of Subatomic and Radiation Physics, Ghent University - UGent, Proeftuinstraat 86, B-9000 Ghent, Belgium

${ }^{b}$ Helmholtz-Institut für Strahlen- und Kernphysik, Bonn University, Nußallee 14-16, D-53115 Bonn, Germany

The Lorentz-covariant Bethe-Salpeter model developed by the Bonn group is used to calculate the electromagnetic form factors of the ground-state hyperons and the helicity amplitudes of the hyperon resonances. The computed magnetic moments of the groundstate hyperons agree well with the experimental values and the magnetic form factors exhibit a dipole $Q^{2}$-dependence. The photo-amplitude of the $\Lambda(1405)$ is badly reproduced, which hints at the special structure of this resonance.

\section{Introduction}

In describing meson electroproduction processes on the nucleon with isobar models, the implementation of electromagnetic (EM) and strong form factors constitutes one of the major sources of uncertainty. This is particularly the case for the electroproduction of kaons from the proton, where the dynamics is the result of a subtle interplay between contributions from multiple nucleon and hyperon $\left(\Lambda^{*}\right)$ resonances. This work focuses on the computation of the EM form factors of the latter. We have used the constituent quark (CQ) model developed by the Bonn group $[1,2]$ to calculate the EM form factors of ground-state hyperons and the helicity amplitudes of hyperon resonances. The Bonn CQ model is based on the Lorentz-covariant Bethe-Salpeter approach and is therefore well suited to describe baryon properties up to high $Q^{2}$, which involve large recoil effects [3].

\section{EM Form Factors in the Bethe-Salpeter Approach}

\subsection{The Bethe-Salpeter Equation}

The Bethe-Salpeter (BS) amplitude $\chi_{\bar{P}}$ is the analogue of the wave function in the Hilbert space of three quarks with Dirac, flavor and color degrees of freedom. Starting from the six-point Green's function, the following momentum-space integral equation for the BS amplitude can be derived :

$\chi_{\bar{P}}=-i G_{0 \bar{P}}\left(K_{\bar{P}}^{(3)}+\bar{K}_{\bar{P}}^{(2)}\right) \chi_{\bar{P}}$.

*e-mail : Tim.VanCauteren@UGent.be 
This expression incorporates all features of the model. It is Lorentz covariant by construction, and the integral kernel is the product of the free three-quark propagator $G_{0 \bar{P}}$ and the sum of all three- and two-particle interactions $K_{\bar{P}}^{(3)}+\bar{K}_{\bar{P}}^{(2)} \cdot G_{0 \bar{P}}$ is approximated by the direct product of three free CQ propagators. We use a linear three-quark confinement potential for $K_{\bar{P}}^{(3)}$ and the 't Hooft instanton induced interaction for $\bar{K}_{\bar{P}}^{(2)}$ [2]. Both interactions are assumed to be instantaneous.

Once the BS amplitudes are known, one can calculate the matrix elements of any operator between two baryon states. When computing electromagnetic form factors, the operator of interest is the electromagnetic current operator. We use the operator $j_{\mu}^{E}=\bar{\Psi} \hat{q} \gamma_{\mu} \Psi$, which describes the photon coupling to a structureless CQ. Here, $\Psi$ and $\bar{\Psi}$ are the CQ destruction and creation operators, and $\hat{q}$ is the CQ charge operator. In the c.o.m. frame of the incoming baryon $\left(\bar{P}^{\prime}=\bar{M}\right)$, the current matrix elements (CME's) are given by :

$$
\begin{gathered}
\left\langle\bar{P}\left|j^{\mu}\right| \bar{M}\right\rangle \simeq-3 \iint \frac{\mathrm{d}^{4}\left[\frac{1}{2}\left(p_{1}-p_{2}\right)\right]}{(2 \pi)^{4}} \frac{\mathrm{d}^{4}\left[\frac{1}{3}\left(p_{1}+p_{2}-2 p_{3}\right)\right]}{(2 \pi)^{4}} \\
\times \bar{\Gamma}_{\bar{P}}^{\Lambda} S_{F}^{1}\left(p_{1}\right) \otimes S_{F}^{2}\left(p_{2}\right) \otimes\left[S_{F}^{3}\left(p_{3}+q\right) \hat{q} \gamma^{\mu} S_{F}^{3}\left(p_{3}\right)\right] \Gamma_{\bar{M}}^{\Lambda},
\end{gathered}
$$

where $\Gamma$ and $\bar{\Gamma}$ are the amputated BS amplitude and its adjoint, and $S_{F}^{i}$ is the $i$ th $\mathrm{CQ}$ propagator $[3,4]$.

\subsection{Form Factors and Helicity Amplitudes}

The electromagnetic properties of composite particles are usually presented in terms of form factors, which are functions of the independent scalars of the system. The most commonly used expression for the spin-1/2 EM-vertex is :

$$
\begin{aligned}
\left\langle B^{\prime}, \bar{P}^{\prime}, \lambda^{\prime}\left|j_{\mu}^{E}(0)\right| B, \bar{P}, \lambda\right\rangle & =e \bar{u}_{\lambda^{\prime}}\left(\bar{P}^{\prime}\right) \Gamma_{\mu} u_{\lambda}(\bar{P}) \\
& =e \bar{u}_{\lambda^{\prime}}\left(\bar{P}^{\prime}\right)\left[\gamma_{\mu} F_{1}^{B^{\prime} B}\left(Q^{2}\right)+\frac{i \sigma_{\mu \nu} q^{\nu}}{2 M_{p}} \kappa_{B^{\prime} B} F_{2}^{B^{\prime} B}\left(Q^{2}\right)\right] u_{\lambda}(\bar{P})
\end{aligned}
$$

where we have introduced the Dirac and Pauli (transition) form factors $F_{1}^{B^{\prime} B}$ and $F_{2}^{B^{\prime} B}$, and the anomalous (transition) magnetic moment $\kappa_{B^{\prime} B}$. Often, the elastic form factors of the ground-state hyperons are expressed in terms of the Sachs' electric and magnetic form factors :

$$
\begin{gathered}
G_{E}^{B}\left(Q^{2}\right)=F_{1}^{B}\left(Q^{2}\right)-\frac{Q^{2}}{4 M_{B} M_{p}} \kappa_{B} F_{2}^{B}\left(Q^{2}\right)=\frac{\left\langle B, \bar{P}^{\prime}, \frac{1}{2}\left|j_{0}^{E}(0)\right| B, \bar{P}, \frac{1}{2}\right\rangle}{\sqrt{4 M_{B}^{2}+Q^{2}}} \\
G_{M}^{B}\left(Q^{2}\right)=F_{1}^{B}\left(Q^{2}\right)+\frac{M_{p}}{M_{B}} \kappa_{B} F_{2}^{B}\left(Q^{2}\right)=\frac{\left\langle B, \bar{P}^{\prime}, \frac{1}{2}\left|j_{+}^{E}(0)\right| B, \bar{P},-\frac{1}{2}\right\rangle}{2 \sqrt{Q^{2}}} .
\end{gathered}
$$

The response of hyperon resonances to the absorption of virtual photons, $\gamma^{*}+Y^{*}\left(M^{*}\right) \rightarrow$ $Y(M)$, is commonly expressed in terms of helicity amplitudes. These are directly proportional to the spin-flip $\left(A_{1 / 2}\right.$ and $\left.A_{3 / 2}\right)$ and non-spin-flip $\left(C_{1 / 2}\right)$ CME's, with proportionality constant $\sqrt{\frac{\pi \alpha}{2 M^{*}\left(M^{* 2}-M^{2}\right)}}$, where $\alpha$ is the fine-structure constant. 


\section{Results and Conclusions}

Table 1

Static electromagnetic properties of the ground-state hyperons. Magnetic moments are expressed in units of $\mu_{N}$, square radii in units of $\mathrm{fm}^{2}$.

\begin{tabular}{ccccc}
\hline $\mathrm{Y}$ & $\mu_{Y}^{\exp }[5]$ & $\mu_{Y}^{\mathrm{calc}}$ & $\left\langle r_{M}^{2}\right\rangle$ & $\left\langle r_{E}^{2}\right\rangle$ \\
\hline$\Lambda$ & $-0.613 \pm 0.004$ & -0.61 & 0.40 & 0.038 \\
$\Sigma^{+}$ & $2.458 \pm 0.010$ & 2.47 & 0.69 & 0.79 \\
$\Sigma^{0}$ & - & 0.73 & 0.69 & 0.150 \\
$\Sigma^{-}$ & $-1.160 \pm 0.025$ & -0.99 & 0.81 & 0.49 \\
$\left|\Sigma^{0} \rightarrow \Lambda\right|$ & $1.61 \pm 0.08$ & 1.52 & 1.96 & -0.120 \\
$\Xi^{0}$ & $-1.250 \pm 0.014$ & -1.33 & 0.47 & 0.140 \\
$\Xi^{-}$ & $-0.6507 \pm 0.0025$ & -0.57 & 0.38 & 0.47 \\
\hline
\end{tabular}

In Tables 1 and 2, we summarize the predictions for the static properties of the groundstate hyperons and resonances respectively. The magnetic moments are generally in very good agreement with the data. The electric mean-square radius of the $\Sigma^{-}$is in agreement with the values of $0.91 \pm 0.32 \pm 0.40 \mathrm{fm}^{2}$ of Adamovich et al. [6] and $0.61 \pm 0.12 \pm 0.09 \mathrm{fm}^{2}$ from Eschrich et al. [7]. The decay widths $\Gamma$ of the hyperon resonances ( $Y^{*}$ 's) are poorly known. From Table 2 it is clear that for the $\Lambda(1405)$, the decay width is overestimated by more than one order of magnitude in our model. This is another indication for the peculiar structure of this resonance, which was recently already alluded to in other hadron models $[8,9]$. More data on the decays of hyperon resonances would clearly help in further identifying the structure of $Y^{*}$ 's.

The elastic Sachs' electric and magnetic form factors of the ground-state hyperons, as well as the transition Dirac and Pauli form factors of the $\Sigma^{0} \rightarrow \Lambda$ transition, are presented in Ref. [4]. There it is shown that the computed magnetic form factors can be nicely described by means of a dipole $G\left(Q^{2}\right) \sim\left(1+\frac{Q^{2}}{\Lambda^{2}}\right)^{-2}$ with cutoffs $\Lambda$ ranging from 0.79 to $1.14 \mathrm{GeV}$. We also predicted that some electric form factors change sign at a finite value of $Q^{2}$.

Table 2

Static electromagnetic properties of the hyperon resonances for which experimental results are available. Photo-amplitudes are expressed in units of $10^{-3} \mathrm{GeV}^{-1 / 2}$ and widths in units of $\mathrm{MeV}$.

\begin{tabular}{ccccc}
\hline$Y^{*}$ & $\left|A_{1 / 2}\right|$ & $\left|A_{3 / 2}\right|$ & $\Gamma_{\text {calc }}$ & $\Gamma_{\exp }[5]$ \\
\hline $\mathrm{P}_{13}(1385)$ & 62.8 & 108 & 1.46 & $0-13.9$ \\
$\mathrm{~S}_{01}(1405)$ & 51.5 & - & 0.912 & $0.019-0.035$ \\
$\mathrm{D}_{03}(1520)$ & 5.50 & 41.2 & 0.258 & $0.0876-0.166$ \\
\hline
\end{tabular}

The results for the helicity amplitudes of the spin $J=1 / 2 \Lambda^{*}$ resonances are displayed in 
Fig. 1. For the $\mathrm{P}_{01}(1600)$, the $A_{1 / 2}$ reaches a maximum at a finite value of $Q^{2}$. Accordingly, our results indicate that resonances which are of minor importance in photoproduction reactions can play a major role in the corresponding electroproduction process. This also holds true for $\Lambda$ resonances with higher spins [10].
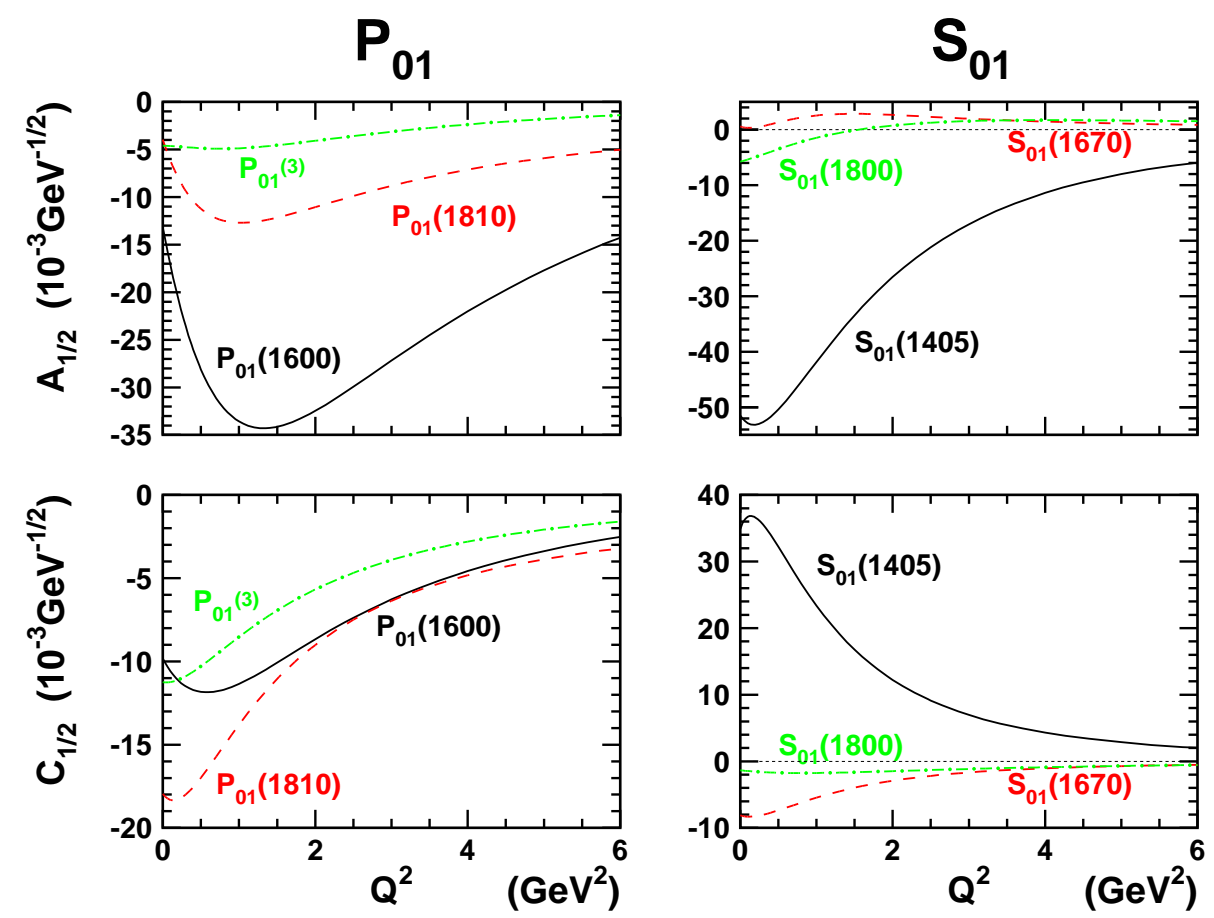

Figure 1. The $Q^{2}$ dependence of the helicity amplitudes for the three lowest-lying $(J=$ $1 / 2, S=-1, T=0) \Lambda^{*}$-baryons with positive parity (left panels) and negative parity (right panels).

\section{REFERENCES}

1. U. Löring, K. Kretzschmar, B. Metsch and H.-R. Petry, Eur. Phys. J. A10 (2001) 309.

2. U. Löring, B. Metsch and H.-R. Petry, Eur. Phys. J. A10 (2001) 395.

3. D. Merten, U. Löring, K. Kretzschmar, B. Metsch and H.-R. Petry, Eur. Phys. J. A14 (2002) 477.

4. T. Van Cauteren, D. Merten, J. Ryckebusch, T. Corthals, S. Janssen, B. Metsch, H.-R. Petry, Eur. Phys. J. A20 (2004) 283.

5. S. Eidelman et al., Phys. Lett. B592 (2004) 1.

6. M. I. Adamovich et al., Eur. Phys. J. C8 (1999) 59.

7. I. Eschrich et al. (SELEX Collaboration), Phys. Lett. B522, No.3-4 (2001) 233.

8. D. Jido, J. A. Oller, E. Oset, A. Ramos and U.-G. Meißner, these proceedings.

9. E. E. Kolomeitsev and M. F. M. Lutz, Phys. Lett. B585 (2004) 243.

10. T. Van Cauteren et al., in preparation. 